\title{
Case Series of Lens Dislocation due to Family Violence
}

\author{
Machiko Shimmura-Tomita Hiroko Takano Yoshiaki Tanaka Rina Takagi \\ Toshikatsu Kaburaki Akihiro Kakehashi \\ Department of Ophthalmology, Saitama Medical Center, Jichi Medical University, Saitama, \\ Japan
}

\section{Keywords}

Lens dislocation · Family violence $\cdot$ Intraocular lens dislocation · Ocular trauma

\begin{abstract}
Differential diagnosis of lens dislocation includes various ocular and systemic diseases, as well as a history of trauma. The purpose of this study is to report cases of lens dislocation caused by family violence, a social problem that is increasing worldwide. Case 1: a 70-year-old female with narrow anterior chamber and high intraocular pressure in her left eye due to lens dislocation was referred to our hospital after her husband had beaten her with a fist. She explained to the previous doctor that she had hit her eye by herself. Case 2: a 99-year-old female with in-the-bag intraocular lens $(\mathrm{IOL})$ dislocation in her left eye 10 years after receiving cataract surgery was referred to our hospital. The following year, she was referred to our hospital because the same incident occurred in her right eye. She explained to the previous doctor that she had fallen but was found to be due to family violence. Case 3: a 62-year-old female suffered dislocation of an IOL inserted in her left eye 10 years previously. While her explanation to the referring doctor was that she tumbled and fell, further inquiry revealed family violence to be the cause. In conclusion, lens dislocation may be caused by family violence despite a conflicting initial clinical history.
\end{abstract}

\section{Introduction}

Incidence of intraocular lens (IOL) dislocation is increasing along with the number of cataract surgery performed each year [1]. The cumulative risk of IOL dislocation is increasing yearly after cataract extraction in a population-based cohort [2]. Differential diagnosis and

\section{Karger ${ }^{\prime \prime}=$}


causes of lens or IOL dislocation include various ocular and systemic diseases. These include pseudo exfoliation syndrome, trauma, high myopia, pigmentary retinal dystrophy, medical history of vitrectomy, and ciliary zonule fragility $[3,4]$. Among them, trauma is often ranked relatively high in reported studies (5.3-16\%) $[1,3,4]$. However, there are few reports with details of the cause of trauma and lens or IOL dislocation.

On the other hand, there are few reports of lens or IOL dislocation due to family violence $[5,6]$. Family violence includes domestic violence, child abuse, and elder abuse. The victims of family violence are increasing yearly around the world because cases are becoming more recognized [7]. Therefore, for example, the Family Violence Prevention and Services Act was enacted since 1984 in the United States. The United Nations General Assembly in 1993 adopted the Declaration on the Elimination of Violence against Women. Like many other countries, the Act on the Prevention of Spousal Violence and the Protection of Victims and the Elderly Abuse Prevention Law was enforced in 2001 and 2006 in Japan.

The purpose of this study is to report details on cases of lens or IOL dislocation caused by family violence. These cases had no other risk factors that could cause lens dislocation.

\section{Case Report}

\section{Case 1}

Patient 1 is a 70-year-old female who was referred to us as angle-closure glaucoma because of narrow anterior chamber and high intraocular pressure in her left eye (Fig. 1a). She explained to a previous doctor that her elbow had struck her left eye and she had monocular diplopia in her left eye 1 week after the incidence. Her BCVA was 0.5, and her IOP was $34 \mathrm{~mm} \mathrm{Hg}$ in her left eye. In addition, the refraction of her left eye was more myopic ( -3.0 Diopter) than her right eye ( -0.75 Diopter). After mydriasis, we found that the narrow anterior chamber was due to lens dislocation (Fig. 1b). Her crystalline lens had mild cortical opacity. There was mild anterior chamber inflammation, but no iris damage. Her optic disc was within normal limits. There was some vitreous opacification in her peripheral fundus, but no damage to her retina. We underwent her interview again in detail when her husband was not present. Finally, she explained that her husband beat her left eye 8 months ago and had hit her left eye again 2 weeks ago. She was treated by the previous doctor as subconjunctival hemorrhage and iritis of unknown cause after the first beating. After she became aware of double vision 1 week after the second beating, she consulted the same doctor again. We promptly performed phacoemulsification and aspiration, pars plana vitrectomy with lens capsule resection, and ciliary sulcus fixation of posterior chamber IOL with 9-0 polypropylene sutures. The eye has been stable since then, and we reintroduced her to the former doctor after 1-month follow-up. At that time, we made her husband promise not to commit family violence again. However, 1 year later, she was introduced to us again with increased intraocular pressure and iritis. The cause of iritis could not be identified as traumatic. She said she hadn't been beaten by her husband. However, we could not stop the steroid eye drops because of recurrence. Intraocular pressure was finally controlled within the normal range by using three types of antiglaucoma instillation. Her visual field was kept within normal limits. Her BCVA in the left eye was 20/16 and IOP was $16 \mathrm{~mm} \mathrm{Hg}$ at the last visit.

Case 2

Patient 2 is a 99-year-old female who was referred to us with complete in-the-bag IOL dislocation into the vitreous cavity in her left eye after she fell, according to her family's explanation (Fig. 1c). She underwent uneventful phacoemulsification cataract extraction and

\section{Karger'}



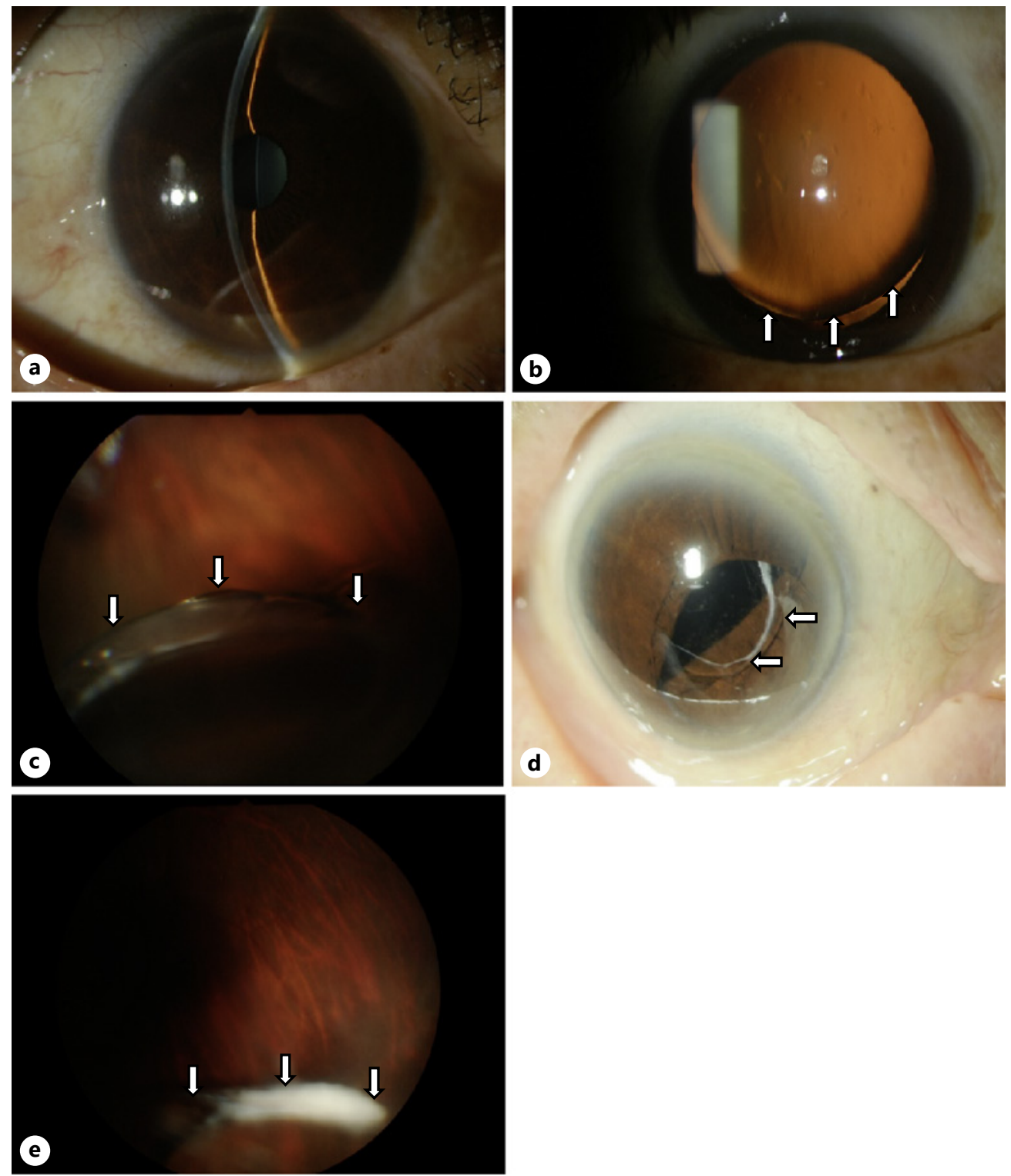

Fig. 1. Slit-lamp photographs of cases. Narrow anterior chamber of case 1 that was due to lens dislocation (shown in $\mathbf{a}, \mathbf{b}$ ). Complete in-the-bag IOL dislocation into the vitreous cavity in the left eye of case 2 (shown in c). In-the-bag IOL dislocation in her right eye approximately 1 year after left eye dislocation (shown in d). Complete in-the-bag IOL dislocation into the vitreous cavity of case 3 (shown in e).

posterior chamber in-the-bag IOL implantation in both eyes when she was 89 years old. However, IOL dislocation was found in the left eye 10 years after the surgery, and we immediately performed IOL removal, pars plana vitrectomy, and ciliary sulcus fixation of posterior chamber IOL with 9-0 polypropylene sutures. There were no abnormal findings in her anterior chamber, angle, vitreous, and retina. At the postoperative outpatient clinic, there was a bruise in the eyelid and forehead of the patient, and elder abuse was discovered after a detailed interview with the key person, her granddaughter. We told her the dangers of violence against the eyes and asked her family to stop the violence. However, she told us that her family relationship was difficult and it was difficult to get the consent of her entire family. Moreover, she did not want police or welfare office intervention. 
Approximately 1 year later, she was referred to us with in-the-bag IOL dislocation in her right eye (Fig. 1d). She had mild iritis. There were no abnormal findings in her fundus. She explained to the previous doctor that she had fallen, but her family explained that one of the family members may have hit her near the eye a few days ago. We immediately performed IOL removal, ciliary sulcus fixation of posterior chamber IOL with 9-0 polypropylene sutures, and pars plana vitrectomy. Both eyes showed a fair prognosis. Her BCVA was 20/125 in the right eye and 20/50 in the left eye; her IOP was $9 \mathrm{~mm} \mathrm{Hg}$ in both eyes at the last visit.

Case 3

Patient 3 is a 62 -year-old female who was referred to us with complete in-the-bag IOL dislocation into the vitreous cavity in her left eye (Fig. 1e). She received uneventful phacoemulsification cataract extraction and posterior chamber in-the-bag IOL implantation in her left eye 10 years prior. Her visual acuity had declined after her husband hit her left eye, but she explained to the previous doctor that she had tumbled and fallen down. When we asked them again what the real reason was, since it was important to choose a treatment policy, her husband confessed to the beating. At that time, we made him promise not to use violence again in her eyes. Her BCVA was 20/16 with +6.5 Diopter in her left eye. Her IOP was $13 \mathrm{~mm}$ Hg. She had mild iritis. There were no abnormal findings in her fundus. We immediately performed IOL removal, ciliary sulcus fixation of posterior chamber IOL with 9-0 polypropylene sutures, and pars plana vitrectomy in her left eye. Her left eye showed good course. Her BCVA was 20/16 and IOP was $13 \mathrm{~mm} \mathrm{Hg}$ at the last visit.

\section{Discussion}

We described 3 patients with IOL or lens dislocation that could be clearly linked to family violence. All cases in this study were female. There are many reports showing that males are more common in IOL dislocation cases in general $[1,3,4]$. When combined with this study and the previous reports, family violence-related IOL or lens dislocation cases were 1 male and 4 females $[5,6]$. It is noteworthy that family violence-related IOL dislocation is clearly higher in females. Generally, females are more prone to become victims of family violence [8]. In addition, it was reported that orbital fracture was the result of sexual assault or domestic violence in one-third of the female patients, but no male patients within 54 consecutive cases of orbital fractures [9]. Thus, as shown in the current case series, female lens dislocation cases without obvious risk factors for lens dislocation should be considered to be possibly due to family violence.

All of the patients in this study did not complain of family violence voluntarily at the first visit. In addition, they were not recognized as family violence victims at the initial examination in former clinics. Patients tend to hide the fact that they are victims of family violence [10]. There are some reports that most abused women did not volunteer a history of violence even to their regular clinicians [11]. Doctors lack knowledge about family violence and lack of education for how to deal with family violence [12]. Our cases may only be the tip of the iceberg. To clarify the possibility of family violence, it is necessary to take a detailed interview in the cases with IOL dislocation without any risk factors.

Case 2 raised an important issue. We could not avoid IOL dislocation occurring in the fellow eye 1 year after IOL dislocation in her left eye. In the other 2 cases, each husband promised not to commit violence in the future, however, we could not confirm if the violence had actually ceased. In fact, it is difficult for ophthalmologists alone to deal with family violence. To prevent recurrence, when an ophthalmologist diagnoses family violence, it may be better to ask a specialized team of family violence for cooperation.

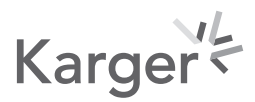


The lens may be dislocated anteriorly or posteriorly, but no lens dislocation to the anterior chamber was observed in our 3 cases. In Cases 2 and 3, the IOL was dislocated into the vitreous cavity, and therefore, a posterior dislocation. In Case 1, IOP elevation and narrow angle were observed, which can be considered as anterior lens dislocation. Under such circumstances, the anterior dislocation in Case 1 may be related to being beaten twice in a relatively short period of time. It was not clear how many days ago Case 2 and 3 were beaten. We suspect that routine abuse may have led to ciliary zonule fragility and lens posterior dislocation in these cases.

On the other hand, the most common sites of injury due to family violence were reported to be the eyes, the side of the face, and the throat or neck [13]. Of ocular injuries in battered women, $86 \%$ were result of a punch with a closed fist, and orbital fracture, ruptured globe, and traumatic hyphema were reported as serious injuries [14]. Family violence and sexual assault were reported as frequent cause of orbital fractures among women [9]. This study revealed that family violence against women may cause IOL dislocation. Further studies in a larger number of cases are necessary to clarify actual conditions of eye injury due to family violence. In addition, it is difficult for ophthalmologists alone to deal with family violence, and therefore, it is necessary to request the cooperation of a specialist in family violence countermeasures.

\section{Conclusion}

Family violence should be considered as a cause of lens dislocation despite a conflicting initial clinical history. Detailed inquiry is required to discover the victims of family violence.

\section{Statement of Ethics}

All procedures performed in studies involving human participants were with the 1964 Helsinki Declaration and its later amendments or comparable ethical standards. Written informed consent was obtained from the patients for publication of this case report and any accompanying images. This study protocol was reviewed and approved by the Institutional Review Board of Jichi Medical University, approval number [S21-064].

\section{Conflict of Interest Statement}

The authors have no conflicts of interest to declare.

\section{Funding Sources}

No funding was received to assist with the preparation of this manuscript.

\section{Author Contributions}

M.S.T. and H.T. contributed to the conception of the work. Y.T. and R.T. searched the literature and extracted the data. M.S.T. and A.K. wrote the manuscript. T.K. revised the manuscript and produced the final version. All authors read and approved the final manuscript. 


\section{Data Availability Statement}

All data generated or analyzed during this study are included in this article. Further inquiries can be directed to the corresponding author.

\section{References}

1 Gimbel HV, Condon GP, Kohnen T, Olson RJ, Halkiadakis I. Late in-the-bag intraocular lens dislocation: incidence, prevention, and management. J Cataract Refract Surg. 2005;31(11):2193-204.

2 Dabrowska-Kloda K, Kloda T, Boudiaf S, Jakobsson G, Stenevi U. Incidence and risk factors of late in-the-bag intraocular lens dislocation: evaluation of 140 eyes between 1992 and 2012. J Cataract Refract Surg. 2015; 41(7):1376-82.

3 Fernández-Buenaga R, Alio JL, Pérez-Ardoy AL, Larrosa-Quesada A, Pinilla-Cortés L, Barraquer R, et al. Late in-the-bag intraocular lens dislocation requiring explantation: risk factors and outcomes. Eye. 2013;27(7): 795-801.

4 Hayashi K, Hirata A, Hayashi H. Possible predisposing factors for in-the-bag and out-of-the-bag intraocular lens dislocation and outcomes of intraocular lens exchange surgery. Ophthalmology. 2007;114(5):969-75.

5 Georgalas I, Ladas I, Papacostantinou D, Taliatzis S, Koutsandrea C. Management of crystalline lens dislocation into the anterior chamber in a victim of domestic violence. Clin Exp Optom. 2012;95(1):113-5.

6 Mutoh T, Tien T, Horie M, Matsumoto Y, Chikuda M. Case of bilateral complete posterior dislocation of lens caused by elder abuse. Clin Ophthalmol. 2012;6:261-3.

7 Carrillo R. Overview of international human rights standards and other agreements and responses of the judicial system to violence against women. Int J Gynaecol Obstet. 2002;78 Suppl 1:S15-20.

8 Sheridan DJ, Nash KR. Acute injury patterns of intimate partner violence victims. Trauma Violence Abuse. 2007;8(3):281-9.

9 Hartzell KN, Botek AA, Goldberg SH. Orbital fractures in women due to sexual assault and domestic violence. Ophthalmology. 1996;103(6):953-7.

10 Rhodes KV, Levinson W. Interventions for intimate partner violence against women: clinical applications. JAMA. 2003;289(5):601-5.

11 McCauley J, Yurk RA, Jenckes MW, Ford DE. Inside "Pandora's box": abused women's experiences with clinicians and health services. J Gen Intern Med. 1998;13(8):549-55.

12 Physicians and domestic violence. Ethical considerations. Council on ethical and judicial affairs, American Medical Association. JAMA. 1992;267(23):3190-3.

13 Reijnders UJ, van der Leden ME, de Bruin KH. Injuries due to domestic violence against women: sites on the body, types of injury and the methods of infliction. Ned Tijdschr Geneeskd. 2006;150(8):429-35.

14 Beck SR, Freitag SL, Singer N. Ocular injuries in battered women. Ophthalmology. 1996;103(1):148-51.

\section{Karger ${ }^{\prime}$}

